\title{
El sistema de bachillerato internacional y su enseñanza en idioma inglés como factores claves para la excelencia académica de los bachilleres del Colegio Americano de Guayaquil
}

\author{
The international baccalaureate system and its teaching in English as key factors \\ for the academic excellence of the high school graduates of the Colegio Americano \\ de Guayaquil.
}

DOI: $10.46932 / \mathrm{sfjdv} 2 \mathrm{n} 3-033$

Received in: May 1st, 2021

Accepted in: Jun 30th, 2021

\section{Wagner Cristhian Cañizares Albán}

Ingeniero en Administración de Empresas de la Universidad Ecotec, Master of Business Administration de la Universidad de las Américas Puebla de México; Rector del Instituto Superior Universitario Blue

Hill College de Guayaquil, Ecuador.

E-mail: wcanizares@bluehill.edu.ec

\section{RESUMEN}

El colegio Americano de Guayaquil fundado hace 79 años se caracteriza por ser líder en la enseñanza bilinguie, habiendo graduado bachilleres en las especialidades de físico matemático, químico biólogos, comercio y de acuerdo a las reformas educativas actualmente gradúa bachilleres en ciencias, ciencias empresariales, y ciencias humanísticas. A lo cual debe agregarse que desde 1985 mediante convenio con la Organización de Bachillerato Internacional con sede en Ginebra, Suiza, imparte la educación IB como un agregado académico que ha dado relevancia a los graduados de esta institución, por cuanto además de ser bachilleres de calidad internacional, dominan el inglés como un segundo idioma, lo que permite que tengan acceso para realizar sus estudios superiores en prestigiosas universidades del exterior.

Las autoridades de educación del país han otorgado los permisos correspondientes, autorizando que el Colegio Americano de Guayaquil enseñe su malla curricular de estudios bajo el sistema de IB en idioma inglés, lo cual lo ha convertido en el pionero y líder de este sistema de enseñanza en la región Costa.

Palabras Claves: Bachillerato internacional, excelencia académica, bachiller, calidad, educación.

\begin{abstract}
The Colegio Americano de Guayaquil was founded 79 years ago. It is characterized by being a leader in bilingual education. It has submitted High School Diplomas to many students in the Physical Mathematic Science, Chemical Biology Science and Business specializations. According to the educational reforms, currently it graduates students in Science, Business Science and Humanistic Sciences. It must be added that since 1985, the International Baccalaureate Organization, Geneva, Switzerland, provides IB education as an academic added value, which has given a high relevance to the graduates of this institution since, besides being international high school graduates, they are proficient in English as a second language; this allows them to enter prestigious universities abroad.

The Ecuadorian education authorities have authorized the Colegio Americano to teach the curriculum under the English IB system. In this way, the School has become the pioneer and leader of this education system in the Coast region.
\end{abstract}

Key words: International Baccalaureate, academic excellence, bachelors, quality, education. 


\section{INTRODUCCIÓN}

La investigación engloba determinar si el sistema de bachillerato internacional y su enseñanza en idioma inglés a los bachilleres del Colegio Americano de Guayaquil, son una herramienta de calidad para el éxito en el aprendizaje de las competencias que requieren para su formación como graduados de élite para contribuir al desarrollo socioeconómico del país.

La institución de educación media, se encuentra ubicada en la ciudad de Guayaquil, Ecuador, fundada hace 79 años y ofrece servicios de enseñanza a niños y jóvenes hasta el nivel de bachillerato, siendo pioneros en la región costa del Ecuador desde 1985 incorporando a su modelo de enseñanza el sistema de bachillerato internacional convirtiéndose en uno de los líderes a nivel de país, entregando certificados BI y diplomas BI, que garantizan el acceso de estudiantes a las más prestigiosas universidades de América, Europa y Asia.

En el desarrollo de esta investigación se establece el derecho a la educación como eje fundamental de las naciones empezando con el sistema inicial hasta el bachillerato, y para estandarizar la educación a la enseñanza a nivel mundial, se modelan sistemas como el del Bachillerato Internacional que buscan mejorar la calidad intelectual de los niños y jóvenes a nivel mundial, con la afiliación de colegios públicos y privados al sistema del bachillerato internacional conocido como IB, cuya sede se encuentra en Ginebra, Suiza.

Se analiza el reconocimiento de los estudios de IB dentro del marco legal ecuatoriano, lo que permite al Colegio Americano de Guayaquil ofrecer la oportunidad de obtener el diploma y certificados IB en idioma inglés y español.

\section{DESARROLLO}

La educación es un derecho irrenunciable de todos los seres humanos, en todos los países del mundo como se establece en el artículo 26 de la Declaración Universal de los Derechos Humanos:

\footnotetext{
“Toda persona tiene derecho a la educación. La educación debe ser gratuita, al menos en lo concerniente a la instrucción elemental y fundamental. La instrucción elemental será obligatoria. La instrucción técnica y profesional habrá de ser generalizada; el acceso a los estudios superiores será igual para todos, en función de los méritos respectivos.

La educación tendrá por objeto el pleno desarrollo de la personalidad humana y el fortalecimiento del respeto a los derechos humanos y a las libertades fundamentales; favorecerá la comprensión, la tolerancia y la amistad entre todas las naciones y todos los grupos étnicos o religiosos, y promoverá el desarrollo de las actividades de las Naciones Unidas para el mantenimiento de la paz.

Los padres tendrán derecho preferente a escoger el tipo de educación que habrá de darse a sus hijos". (Organización de las Naciones Unidas, 1948)
} 
Por consiguiente los padres de familia son libres de escoger el sistema para educar a sus hijos, de manera privada o pública, que ha permitido el desarrollo de varios sistemas de educación a nivel internacional, que comprenden el nivel K-12, que significa 12 años de estudios.

En la búsqueda de un sistema de enseñanza de calidad se crea la International Baccalaureate Organization (IBO) que tiene varios programas de certificación que van desde el nivel inicial hasta el bachillerato, como se mencionan en el gráfico siguiente:

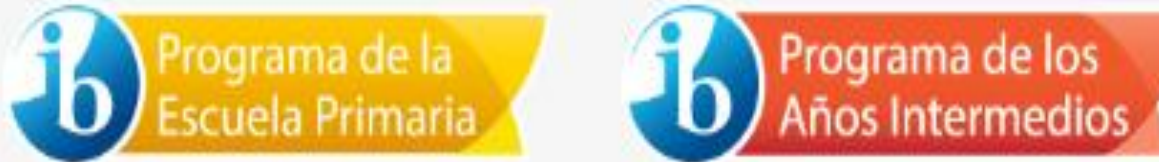

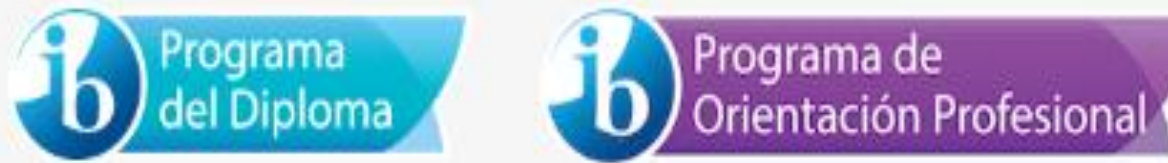

Fuente: (IBO, 2016)

Los objetivos del programa de bachillerato internacional que son liderados por la Organización de Bachillerato Internacional con sede en Ginebra, Suiza; señala que tiene como fin "ofrecer mejores oportunidades que otros programas para formar jóvenes solidarios, informados y ávidos de conocimiento, que se sientan motivados para alcanzar sus metas. Esperamos que nuestros alumnos contribuyan a construir un mundo mejor mediante el entendimiento mutuo y el respeto intercultural". (IBO, 2016)

Este sistema de enseñanza mundial de educación acoge a más de 4.000 colegios en el mundo de carácter público y privado, en los cuales trabajan más de 70.000 educadores, quienes a su vez enseñan a más de un millón de alumnos en todo el mundo. (IBO, 2016)

La opinión generalizada de los estudiantes acerca del programa de IB se centra en los tres pensamientos descritos a continuación, (IBO, 2016):

$\checkmark$ Ayudan a descubrir las fortalezas y debilidades personales

$\checkmark$ Fomentan la confianza en sí mismos y la independencia

$\checkmark$ Enseñan a los alumnos a trabajar de forma más inteligente, en lugar de trabajar más duro

Una vez analizada la educación como un derecho humano al cual se tiene acceso de manera gratuita y de calidad como responsabilidad de los estados, el Ecuador consagra en su artículo 26 que la 
educación "es un derecho de las personas a lo largo de su vida y un deber ineludible e inexcusable del Estado. Constituye un área prioritaria de la política pública y de la inversión estatal, garantía de la igualdad e inclusión social y condición indispensable para el buen vivir”. (Asamblea Nacional Constituyente, 2008)

De esta manera al ser un área prioritaria el accionar del país se enfoca a garantizar su acceso sin discriminación a los niveles

$$
\begin{array}{ll}
\checkmark & \text { Inicial } \\
\checkmark & \text { Básico } \\
\checkmark & \text { Bachillerato } \\
\checkmark & \text { Tercer Nivel }
\end{array}
$$

Así mismo el Ecuador en el artículo 29 de su constitución señala que "las madres y padres o sus representantes tendrán la libertad de escoger para sus hijas e hijos una educación acorde con sus principios, creencias y opciones pedagógicas" (Asamblea Nacional Constituyente, 2008).

Partiendo de este derecho de libertad que asegura la máxima legislación del Ecuador los padres de familia son libres de escoger donde pueden estudiar sus hijos, sea en una institución pública o de carácter privado, para esto los lineamientos de educación son los mismos, sin embargo, se diferencian en calidad académica, docentes, infraestructura, oportunidades de intercambio, y sin dudarlo el bachillerato internacional que es el eje de esta investigación.

La política pública del Ecuador a través del Ministerio de Educación, institución que norma la educación de nivel inicial, básica y de bachillerato en el Ecuador, en el año 2006 firmó un convenio con la IBO, institución con sede en Suiza, para que los colegios del sector público de Ecuador adopten y enseñen bajo los lineamientos del sistema de bachillerato internacional, al igual que los colegios privados.

El reconocimiento de este programa en el territorio ecuatoriano se encuentra establecido en el Acuerdo No. 0224-13 que en el artículo 3 expresa "El Ministerio de Educación reconocerá y aprobará la implementación de la oferta académica y propuestas pedagógicas de Bachillerato Internacional en las instituciones educativas que hayan obtenido la autorización correspondiente por parte de la IBO" (Espinoza, 2013); por lo que claramente las instituciones educativas que se encuentran autorizadas pueden ofertar el programa de diploma de IB y será equivalente al programa de educación ecuatoriano.

El Colegio Americano de Guayaquil, tiene la autorización legal emitida por la IBO y de manera local reconocido por el Ministerio de Educación.

A continuación se muestra el registro obtenido desde la página oficial del IBO en el que se indica que el Colegio Americano de Guayaquil está acreditado para ofertar este programa de IB. 


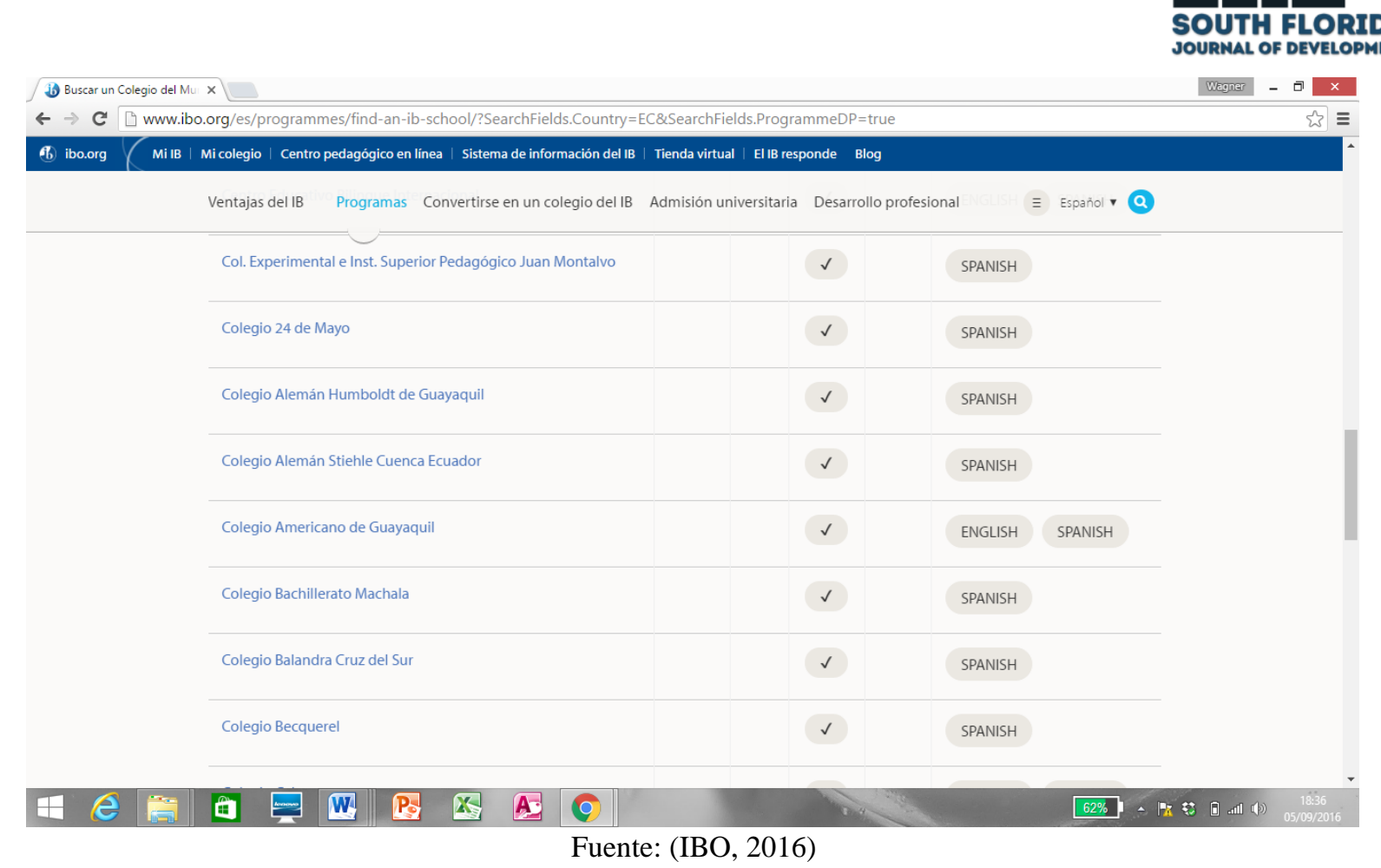

Es importante mencionar que el Colegio Americano de Guayaquil está autorizado de acuerdo al gráfico anterior, a impartir su programa de diploma y certificado de IB en idioma inglés y español, a diferencia de los otros colegios que solo están autorizados a dictarlo en español.

La fortaleza del Colegio Americano desde su fundación hace 79 años es impartir educación bilinguie, esto es, español e inglés, lo cual le ha dado prestigio y lo ha posesionado como la primera institución educativa a nivel de la ciudad de Guayaquil que garantiza que sus graduados dominen en forma completa, el idioma inglés, que a nivel de Ecuador proporciona una ventaja competitiva a los graduados de esta importante organización.

Se realiza una investigación de enfoque cuantitativo con un cuestionario que se adjunta como anexo 1, en el cual se utilizan dos grupos de estudiantes de tercero bachillerato de 20 estudiantes cada uno, para comprobar la veracidad de la hipótesis planteada en la investigación:

La metodología del programa de Bachillerato Internacional y su enseñanza en idioma inglés como factores claves para la excelencia académica de los bachilleres del Colegio Americano de Guayaquil. 


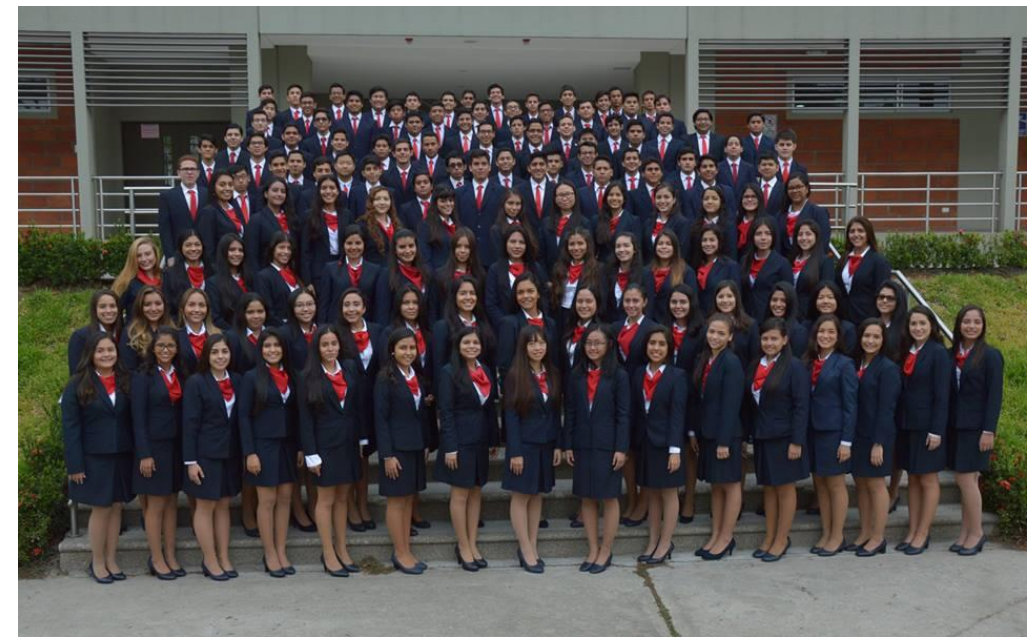

Fuente: (Colegio Americano de Guayaquil, 2016)

\section{ANÁLISIS DE LOS RESULTADOS}

Los resultados obtenidos para la comprobación de la hipótesis determinan que:

El $87.5 \%$ de los estudiantes creen que el bachillerato internacional es la causa de que se gradúen como bachilleres con una formación de calidad.

En relación a la apreciación de los estudiantes si el sistema de educación tradicional cumple con la expectativa de aprendizaje de cada estudiante, se concluye que un $85 \%$ cree que el sistema de enseñanza tradicional no satisface las altas expectativas de aprendizaje que tienen los bachilleres del Colegio Americano de Guayaquil.

La tradición de 79 años de enseñanza del modelo educativo en idioma inglés, ha sido y es un factor diferenciador de los graduados del Colegio Americano; su equivalencia con el programa de bachillerato internacional en inglés, hace que en esta investigación se concluya que los estudiantes encuestados en un $100 \%$ indiquen que el sistema de enseñanza de IB se complementa con una enseñanza total en idioma inglés tales como impartir educación en inglés de las ciencias: language and literatura, history, business and management, geography, economics, physics, environmental systems, biology, chemistry, mathematics, visual arts, las mismas que son dictadas por profesores nativos de habla inglesa con maestrías en sus respectivas especializaciones.

Los estudiantes del Colegio Americano rinden evaluaciones muy exigentes previas a obtener su grado de bachiller nacional y su diploma de IB, habiendo obtenido los primeros lugares en la clasificación general de los colegios del Ecuador, lo cual es el reflejo de la excelente enseñanza que reciben los estudiantes.

Los jóvenes de 16 a 18 años que cursan el último nivel de estudios, en su mayoría han definido las universidades en las que continuarán sus estudios superiores, esto es, que conocen con anticipación de los sistemas de evaluación exigidos para el ingreso a universidades extranjeras y/o nacionales, por lo que un 
87,5\% de los encuestados concluye que el haber estudiado bajo el sistema de IB les permite acceder a la universidad con bases académicas de óptimo nivel.

Estos resultados se ven reflejados en las estadísticas que muestran que los estudiantes del Colegio Americano ingresan sin problemas a las mejores universidades del mundo, especialmente a las universidades de Estados Unidos.

\section{CONCLUSIONES}

Estudiar en el mundo del AMERICANO en la ciudad de Guayaquil es una experiencia diferenciadora para sus estudiantes, que a lo largo de los años han destacado en los distintos sectores de la economía del país, por lo que los estudiantes encuestados creen firmemente que el haber obtenido un diploma IB, los han convertido en un ciudadano con una ventaja competitiva que lo diferencia de los otros bachilleres del Ecuador.

Los estudiantes cuentan con un alto nivel académico que los prepara para rendir pruebas nacionales e internacionales, lo cual está demostrado por el éxito que han obtenido en sus evaluaciones, ocupando en el ranking de colegios del Ecuador los primeros puestos.

En el Colegio Americano de Guayaquil existen otros factores complementarios que son claves para alcanzar una educación de calidad bajo el programa de IB en idioma inglés que entre otros son:

$\checkmark$ Líderes en deportes tales como: beisbol, básquet, voleibol, fútbol, cheerleading, danza, gimnasia olímpica, ajedrez.

$\checkmark$ Profesores extranjeros nativos de Estados Unidos, Inglaterra y Europa.

$\checkmark$ Conciencia ecológica

$\checkmark$ Prestigio

$\checkmark$ Instalaciones, y laboratorios con tecnología de punta

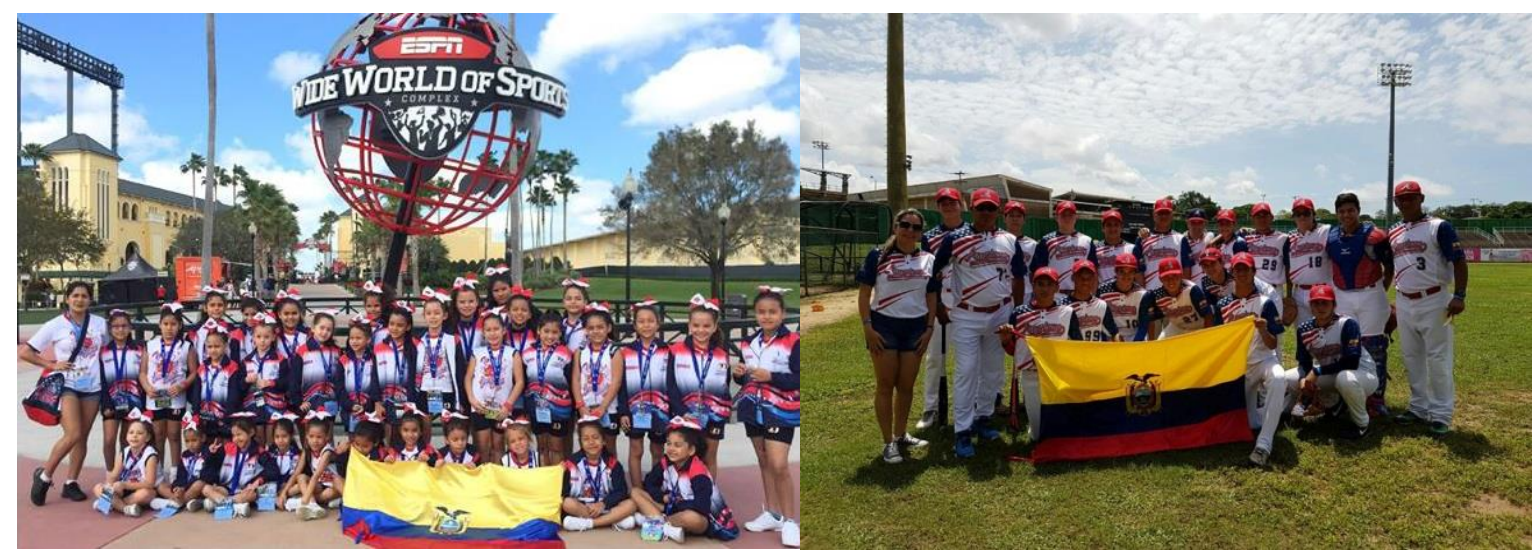

Fuente: autores 


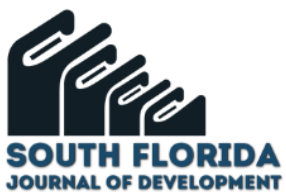

En estas gráficas, se puede apreciar a la delegación del Colegio Americano de Guayaquil que representó al país en el torneo UCA de Cheerleading en Orlando, Florida, obteniendo el primer lugar de los equipos participantes del Ecuador, y la selección de beisbol del Colegio Americano de Guayaquil que representó al país en el torneo Mr. Prospects de Cartagena, Colombia obteniendo una destacada participación.

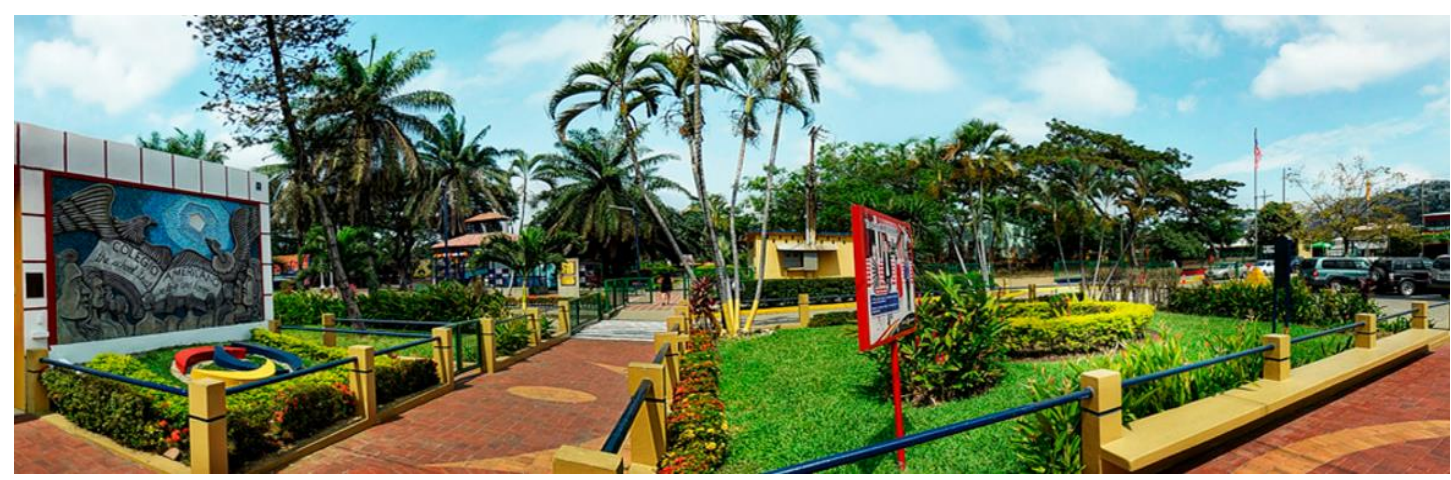

Fuente: (Colegio Americano de Guayaquil, 2016)

Se aprecia el cuidado de los jardines naturales del campus que transmite conciencia ambiental.

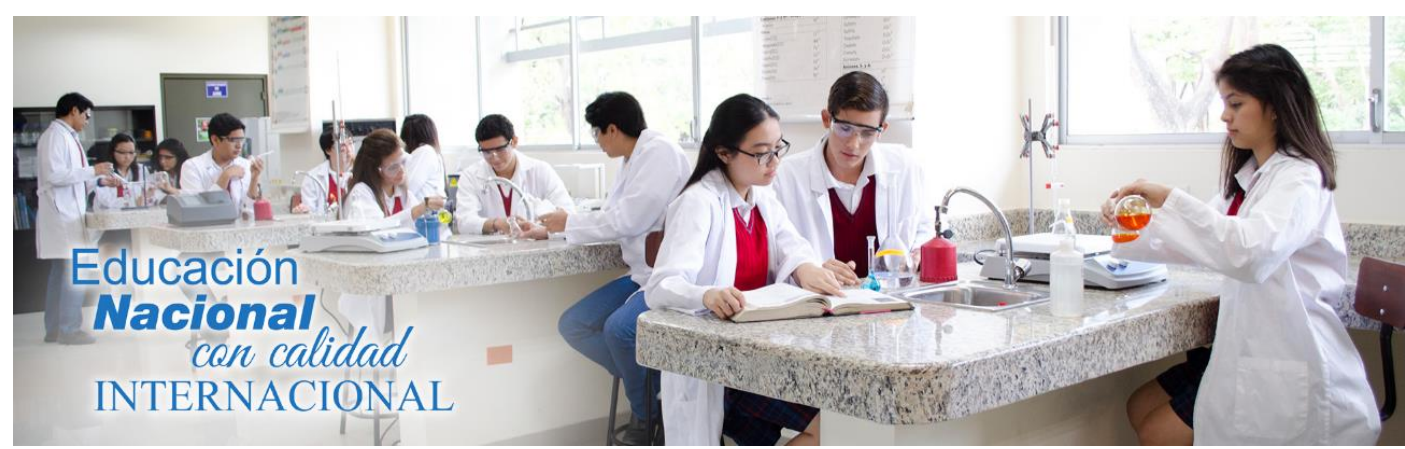

Fuente: (Colegio Americano de Guayaquil, 2016)

En esta gráfica se expone las instalaciones de laboratorios de investigación de química y física que están a disposición de los estudiantes.

De igual manera los estudiantes han determinado beneficios educativos y económicos que tienen a la obtención del diploma y/o certificados del Bachillerato Internacional para los que ingresan a la universidad, estos son:

$\checkmark$ Ahorro económico en estudios universitarios

$\checkmark$ Preparación para exámenes internacionales

$\checkmark$ Nivel académico mundial

$\checkmark$ Mejores oportunidades laborales

$\checkmark$ Titulo con prestigio 
Por lo cual se concluye que un $30 \%$ cree que la preparación para exámenes internacionales es el beneficio más importante; el 20\% el nivel académico mundial; un 17.5\% para ahorro económico en estudios universitarios; $17.5 \%$ para mejores oportunidades laborales; y un 15\% concluye que es un título con prestigio.

Finalmente los estudiantes al haber cursado toda su vida académica en una institución como el Colegio Americano, concluyen en un $82.5 \%$ que las enseñanzas IB en inglés que han recibido han sido de calidad y un $17.5 \%$ que es satisfactoria. Lo importante es que la gran mayoría piensa que ha recibido una excelente educación BI.

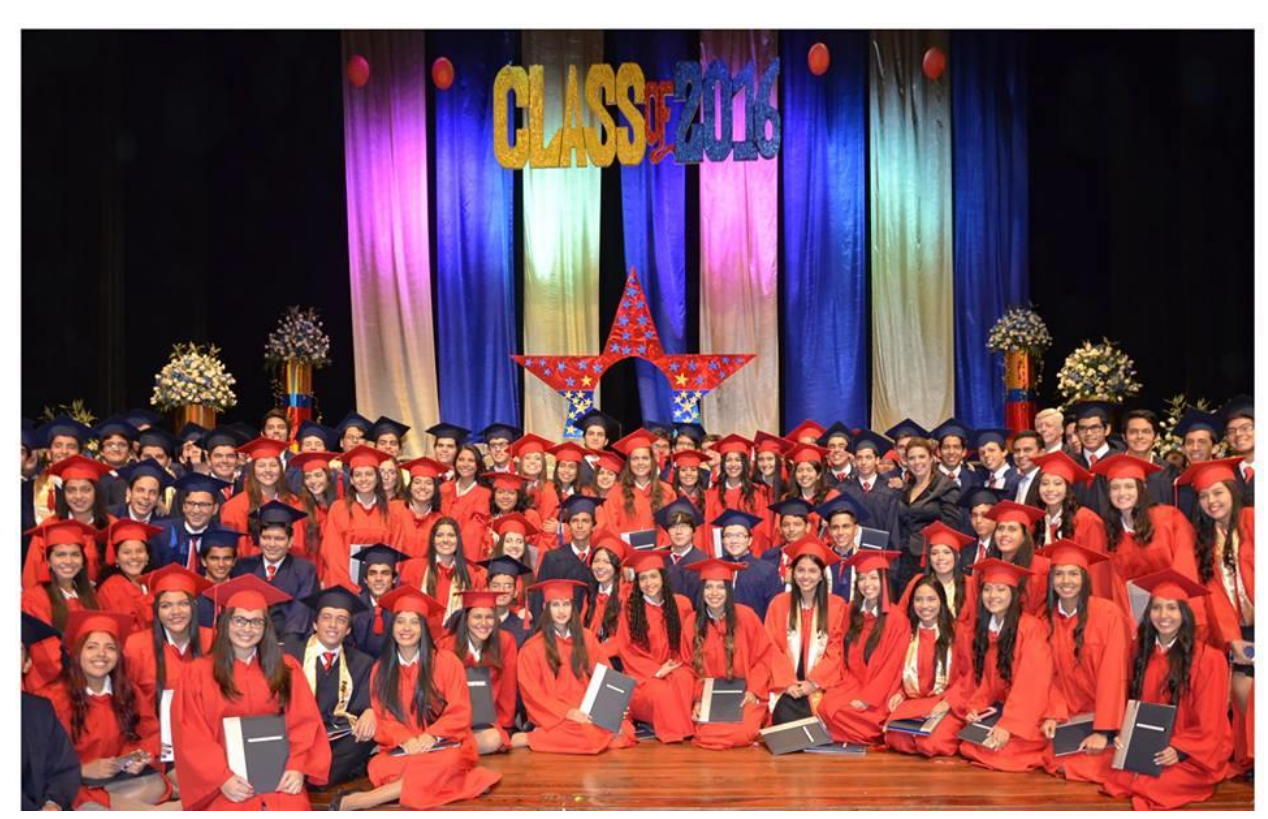

Fuente: (Colegio Americano de Guayaquil, 2016)

En el Ecuador las empresas privadas y las empresas públicas están exigiendo que para llenar sus puestos directivos es necesario que el aspirante domine el idioma inglés, porque las relaciones internacionales del comercio, la industria, la educación, entre otras, exigen profesionales con dominio técnico de especialización en este idioma mundial. 


\section{BIBLIOGRAPHIC REFERENCES}

National Constituent Assembly. (October 20, 2008). Constitution of the Republic of Ecuador. Quito, Ecuador: R.O. 449.

Colegio Americano de Guayaquil. (2016). www.colegioamericano.edu.ec. Retrieved September 02, 2016, from http://colegioamericano.edu.ec/historia/.

Colegio Americano de Guayaquil. (2016). www.colegioamericano.edu.ec. Retrieved September 02, 2016, from http://colegioamericano.edu.ec/wp-content/uploads/2015/09/cuadro-estadistico-01.jpg

Espinoza, A. (July 16, 2013). Acuerdo No. 0224-13. Quito, Ecuador: Minister of Education.

IBO. (2016). www.ibo.org. Retrieved September 03, 2016, from http://www.ibo.org/es/

IBO. (2016). www.ibo.org. Retrieved August 26, 2016, from http://www.ibo.org/es/benefits-of-the-ib/.

IBO. (2016). www.ibo.org. Retrieved August 26, 2016, from http://www.ibo.org/es/benefits-of-the-ib/.

IBO. (2016). www.ibo.org. Retrieved September 04, 2016, from http://www.ibo.org/es/benefits-of-the$\mathrm{ib} /$ student-views-of-the-ib/.

IBO. (2016). www.ibo.org. Retrieved September 01, 2016, from http://www.ibo.org/es/programmes/findan-ib-

school/?SearchFields.Country=EC\&SearchFields.ProgrammeDP=true.

United Nations. (December 10, 1948). Universal declaration of human rights. Paris: United Nations General Assembly. 


\section{ANEXO 1}

\section{CUESTIONARIO}

1. ¿Cree usted que el bachillerato internacional tiene como efecto graduar jóvenes con alto grado de calidad? (Marque con una cruz la respuesta).<smiles></smiles>

2. ¿El sistema de enseñanza tradicional cumple las expectativas de aprendizaje de usted?<smiles>C1CC2CC1C2</smiles>

3. ¿El sistema de enseñanza de IB se complementa con una enseñanza totalmente en el idioma inglés?

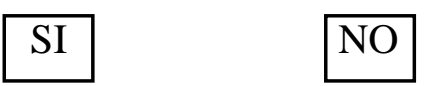

4. ¿El haber estudiado bajo el sistema de IB permite que sus evaluaciones tengan mejores resultados?<smiles>O=NCC1CCC1</smiles>

5. ¿El haber estudiado bajo el sistema de IB permife que al acceder a la universidad esté preparado de una mejor manera?

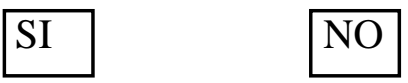

6. ¿Considera usted que al ser un bachiller IB se convierte en un joven con una ventaja diferenciadora del resto de jóvenes?

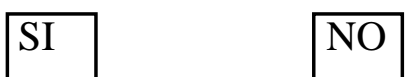

7. ¿Cree usted que su rendimiento las pruebas nacionales será mejor por estudiar en el programa IB?

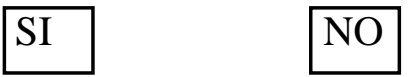

8. ¿Qué otros factores son complementarios para alcanzar una educación de calidad en un programa de IB en el Colegio Americano de Guayaquil?

9. ¿Cuáles son los beneficios educativos y económicos que otorga la obtención del diploma y/o certificados del Bachillerato Internacional para el estudiante que ingresa a la universidad?

10. La enseñanza que ha recibido del Colegio Americano de Guayaquil ha sido:

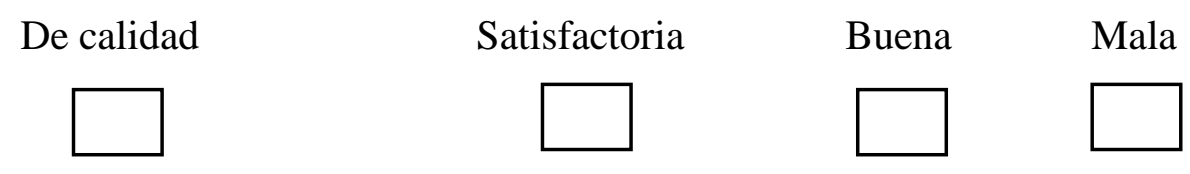

